\title{
Self-similar solutions of some model degenerate partial differential equations of the second, third and fourth order
}

\author{
M. Ruzhansky ${ }^{1,2, *}$ \\ and A. Hasanov ${ }^{3}{ }^{* *}$ \\ (Submitted by A. A. Editor-name) \\ ${ }^{1}$ Department of Mathematics, Analysis, Logic and Discrete Mathematics Ghent University, Belgium \\ ${ }^{2}$ School of Mathematical Sciences, Queen Mary University of London, United Kingdom \\ ${ }^{3}$ Institute of Mathematics, 81 Mirzo-Ulugbek street, Tashkent, 700170 Uzbekistan ${ }^{* * *}$
}

Received July 27, 2020; revised August -, 2020; accepted August 31, 2019

\begin{abstract}
When studying boundary value problems for some partial differential equations arising in applied mathematics, we often have to study the solution of a system of partial differential equations satisfied by hypergeometric functions and find explicit linearly independent solutions for the system. In this study, we construct self-similar solutions of some model degenerate partial differential equations of the second, third, and fourth order. These self-similar solutions are expressed in terms of hypergeometric functions.
\end{abstract}

2010 Mathematical Subject Classification: Primary 35K65; Secondary 33C20, 33C65

Keywords and phrases: PDE of degenerate type; Self-made solutions; Linearly independent solutions, Generalized hypergeometric function, decomposition formulas, Integral representations.

\section{INTRODUCTION AND PRELIMINARIES}

A self-similar solution is a solution to some system or equation in which independent variables do not appear independently, but only in combination. To obtain the desired combination of variables for a self-similar solution one often uses the methods of the theory of dimensions. The methods of the theory of dimensions originate from the works of J. Bertrand J., A. Vaschy, subsequently generalised by H. Weyl.

As an example ([1, p. 113]), we consider the problem of the diffusion of vortices in a viscous incompressible fluid under the assumption that the motion of the fluid is plane-parallel and the fluid occupies the entire plane. The motion in question is transient. Suppose that at the initial moment of time the fluid can potentially move everywhere, except for the pole, which is a trace on the plane of motion of an infinite rectilinear concentrated vortex with circulation. The equation of vortex propagation in this case has the form

$$
\frac{\partial \Omega}{\partial t}=\nu\left(\frac{\partial^{2} \Omega}{\partial r^{2}}+\frac{1}{r} \frac{\partial \Omega}{\partial r}\right)
$$

where $\Omega$ is the angular velocity of the fluid particles in concentrated circles, and $\nu$ is the coefficient of kinematic viscosity of the fluid. The solution is sought in the form

$$
\Omega(r, t)=\frac{E}{\nu t} \psi(\xi), \xi=\frac{r^{2}}{\nu t} \text {. }
$$

* E-mail: michael.ruzhansky@ugent.be, ruzhansky@gmail.com

** E-mail: anvarhasanov@yahoo.com

*** The authors were supported by the FWO Odysseus 1 grant G.0H94.18N: Analysis and Partial Differential Equations. M.R. was also supported by the EPSRC Grant EP/R003025/1 and by the Leverhulme Research Grant RPG-2017-151. 
Substituting (2) into equation (1), we obtain the solution

$$
\Omega(r, t)=\frac{E}{\nu t} A e^{-\frac{r^{2}}{4 \nu t}},
$$

where $A$ is determined from the initial condition of the problem. Thus, (3) is a self-similar solution to equation (1).

It is known that to solve applied problems, one needs to set up a mathematical model of the problem under consideration. In many mathematical models, degenerate differential equations appear (especially in gas dynamics, quantum chemistry, in theoretical physics, in the theory of infinitesimal bending of surfaces of revolution, a momentless theory of shells, etc.).

L.D. Landau and E.M. Lifshits in their article [2] explored the features of the shock wave flow using the Euler-Tricomi equation

$$
u_{x x}+u_{y y}+\frac{1}{3 y} u_{y}=0,
$$

and defined particular solutions of the form

$$
u_{k}=x^{2 k} F\left(-k,-k+\frac{1}{2} ;-2 k+\frac{5}{6} ; 1+\frac{4 y^{2}}{9 x^{2}}\right),
$$

where $k= \pm \frac{n}{2}, \frac{1}{3} \pm \frac{n}{2}, n \in N_{0}$.

Note that the energy absorbed by a non-ferromagnetic conducting sphere placed in an external inhomogeneous magnetic field is calculated explicitly using the hypergeometric functions of many variables $([3])$.

The hypergeometric functions of Kampe de Feriet also appear in theoretical physics and quantum chemistry (see e.g. [4]). In the monographs [5] - [7], attention was drawn to the fact that many problems of supersonic gas dynamics are solved using hypergeometric functions.

Using the method of self-similar solutions in articles [8] - [20], fundamental solutions were found and in articles [21] - [24] the main boundary-value problems for the generalised axisymmetric Helmholtz equation were solved.

In this paper, using the method of self-similar solutions, we construct some special solutions of degenerate partial differential equations that are expressed by hypergeometric functions.

\section{A PARABOLIC EQUATION WITH ONE LINE OF DEGENERATION}

Consider in the domain $\Omega=\{(x, t): x>0, t>0\}$, the degenerate parabolic equation

$$
L u \equiv u_{t}-u_{x x}-\frac{2 \alpha}{x} u_{x}=0, \alpha=\text { const }>0 .
$$

We seek self-similar solutions of equation (4) in the form

$$
u=P \omega(\sigma),
$$

where $\omega=\omega(\sigma)$ is an unknown function, and where $\sigma=-\frac{x^{2}}{4 t}, P=t^{-\frac{1}{2}}$. Substituting (5) into equation (4), we have

$$
P \omega_{\sigma \sigma} \sigma_{x}^{2}+\left[2 P_{x} \sigma_{x}+P\left(\sigma_{x x}+\frac{2 \alpha}{x} \sigma_{x}-\sigma_{t}\right)\right] \omega_{\sigma}+\left(P_{x x}+\frac{2 \alpha}{x} P_{x}-P_{t}\right) \omega=0 .
$$

After elementary calculations, we find

$$
\begin{gathered}
\sigma_{x}^{2}=-\frac{1}{t} \sigma, 2 P_{x} \sigma_{x}+P\left(\sigma_{x x}+\frac{2 \alpha}{x} \sigma_{x}-\sigma_{t}\right)=-P \frac{1}{t}\left(\frac{1+2 \alpha}{2}-\sigma\right), \\
P_{x x}+\frac{2 \alpha}{x} P_{x}-P_{t}=\frac{1}{2} P \frac{1}{t} .
\end{gathered}
$$


Therefore, in view of the indicated equalities, the ordinary differential equation (6) has the form

$$
\sigma \omega_{\sigma \sigma}+\left(\frac{1+2 \alpha}{2}-\sigma\right) \omega_{\sigma}-\frac{1}{2} \omega=0
$$

It is known $([25])$ that the equation

$$
x w_{x x}+(c-x) w_{x}-a w=0,
$$

has two linearly independent solutions

$$
\begin{aligned}
& w_{1}=c_{11} F_{1}(a ; c ; x)=c_{1} e^{x}{ }_{1} F_{1}(c-a ; c ;-x), \\
& w_{2}=c_{2} x^{1-c}{ }_{1} F_{1}(a-c+1 ; 2-c ; x)=c_{2} x^{1-c} e^{x}{ }_{1} F_{1}(1-a ; 2-c ;-x),
\end{aligned}
$$

where the hypergeometric function ${ }_{1} F_{1}(a ; c ; x)$ has the form

$$
{ }_{1} F_{1}(a ; c ; x)=\sum_{m=0}^{\infty} \frac{(a)_{m}}{(c)_{m} m !} x^{m},
$$

and

$$
(a)_{m}=\Gamma(a+m) / \Gamma(a)=a(a+1)(a+2) \cdots(a+m-1)
$$

is the Pochhammer symbol ([25]). Therefore, taking into account (9) and (7), we define

$$
\omega_{1}=c_{11} F_{1}\left(\frac{1}{2} ; \frac{1+2 \alpha}{2} ; \sigma\right), \omega_{2}=c_{2} \sigma^{\frac{1-2 \alpha}{2}}{ }_{1} F_{1}\left(1-\alpha ; \frac{3-2 \alpha}{2} ; \sigma\right) .
$$

Substituting (10) into (5), we finally obtain

$$
\begin{gathered}
u_{1}(x, t)=c_{1} \frac{1}{\sqrt{t}}{ }_{1} F_{1}\left(\frac{1}{2} ; \frac{1+2 \alpha}{2} ;-\frac{x^{2}}{4 t}\right), \\
u_{2}(x, t)=c_{2} \frac{1}{\sqrt{t}}\left(\frac{x^{2}}{4 t}\right)^{\frac{1-2 \alpha}{2}}{ }_{1} F_{1}\left(1-\alpha ; \frac{3-2 \alpha}{2} ;-\frac{x^{2}}{4 t}\right),
\end{gathered}
$$

two self-similar solutions of equation (4), where $c_{1}, c_{2}$ are constants. Note that in [26], the boundary value problems for the equation $L^{m} u=0$ were considered.

\section{A PARABOLIC EQUATION WITH TWO LINES OF DEGENERACY}

In the domain $\Omega=\{(x, y, t): x>0, y>0, t>0\}$, we consider the equation

$$
L u \equiv u_{t}-u_{x x}-u_{y y}-\frac{2 \alpha}{x} u_{x}-\frac{2 \beta}{y} u_{y}=0, \alpha, \beta=\text { const. }
$$

The solution to equation (13) is sought in the form

$$
u=P \omega(\xi, \eta),
$$

where $\xi=-\frac{x^{2}}{8 t}, \eta=-\frac{y^{2}}{8 t}, P=t^{-\frac{1}{2}}$, and $\omega(\xi, \eta)$ is an unknown function. Substituting (14) into (13), we have

$$
A_{1} \omega_{\xi \xi}+A_{2} \omega_{\xi \eta}+A_{3} \omega_{\eta \eta}+A_{4} \omega_{\xi}+A_{5} \omega_{\eta}+A_{6} \omega=0
$$


where

$$
\begin{aligned}
& A_{1}=P\left(\xi_{x}^{2}+\xi_{y}^{2}\right), \quad A_{2}=2 P\left(\xi_{x} \eta_{x}+\xi_{y} \eta_{y}\right), A_{3}=P\left(\eta_{x}^{2}+\eta_{y}^{2}\right), \\
& A_{4}=\frac{2 \alpha}{x} P \xi_{x}+\frac{2 \beta}{y} P \xi_{y}-P \xi_{t}+2 P_{x} \xi_{x}+P \xi_{x x}+2 P_{y} \xi_{y}+P \xi_{y y} \\
& A_{5}=\frac{2 \alpha}{x} P \eta_{x}+\frac{2 \beta}{y} P \eta_{y}-P \eta_{t}+2 P_{x} \eta_{x}+P \eta_{x x}+2 P_{y} \eta_{y}+P \eta_{y y} \\
& A_{6}=-P_{t}+P_{x x}+P_{y y}+\frac{2 \alpha}{x} P_{x}+\frac{2 \beta}{y} P_{y} .
\end{aligned}
$$

Calculating the values of the coefficients of equation (15), we obtain a system of partial differential equations

$$
\left\{\begin{array}{c}
\xi \omega_{\xi \xi}+\left(\frac{1+2 \alpha}{2}-\xi\right) \omega_{\xi}-\eta \omega_{\eta}-\frac{1}{2} \omega=0 \\
\eta \omega_{\eta \eta}+\left(\frac{1+2 \beta}{2}-\eta\right) \omega_{\eta}-\xi \omega_{\xi}-\frac{1}{2} \omega=0 .
\end{array}\right.
$$

In the monograph [25], [27] the following system of hypergeometric equations was considered

$$
\left\{\begin{array}{l}
x w_{x x}+\left(c_{1}-x\right) w_{x}-y w_{y}-a w=0 \\
y w_{y y}+\left(c_{2}-y\right) w_{y}-x w_{x}-a w=0
\end{array}\right.
$$

and 4 linearly independent solutions were found, expressed in terms of the confluent Kummer functions,

$$
\begin{gathered}
w_{1}=\lambda_{1} \Psi_{2}\left(a ; c_{1}, c_{2} ; x, y\right), \\
w_{2}=\lambda_{2} x^{1-c_{1}} \Psi_{2}\left(a+1-c_{1} ; 2-c_{1}, c_{2} ; x, y\right), \\
w_{3}=\lambda_{3} y^{1-c_{2}} \Psi_{2}\left(a+1-c_{2} ; c_{1}, 2-c_{2} ; x, y\right), \\
w_{4}=\lambda_{4} x^{1-c_{1}} y^{1-c_{2}} \Psi_{2}\left(a+2-c_{1}-c_{2} ; 2-c_{1}, 2-c_{2} ; x, y\right),
\end{gathered}
$$

where $\lambda_{i}=$ const, $i=1,2,3,4$, and

$$
\Psi_{2}\left(a ; c_{1}, c_{2} ; x, y\right)=\sum_{m, n=0}^{\infty} \frac{(a)_{m+n}}{\left(c_{1}\right)_{m}\left(c_{2}\right)_{n} m ! n !} x^{m} y^{n} .
$$

In view of (18) - (21), we define

$$
\begin{gathered}
\omega_{1}=\lambda_{1} \Psi_{2}\left(\frac{1}{2} ; \frac{1+2 \alpha}{2}, \frac{1+2 \beta}{2} ;-\frac{x^{2}}{8 t},-\frac{y^{2}}{8 t}\right), \\
\omega_{2}=\lambda_{2}\left(\frac{x^{2}}{8 t}\right)^{\frac{1-2 \alpha}{2}} \Psi_{2}\left(1-\alpha ; \frac{3-2 \alpha}{2}, \frac{1+2 \beta}{2} ;-\frac{x^{2}}{8 t},-\frac{y^{2}}{8 t}\right), \\
\omega_{3}=\lambda_{3}\left(\frac{y^{2}}{8 t}\right)^{\frac{1-2 \beta}{2}} \Psi_{2}\left(1-\beta ; \frac{1+2 \alpha}{2}, \frac{3-2 \beta}{2} ;-\frac{x^{2}}{8 t},-\frac{y^{2}}{8 t}\right), \\
\omega_{4}=\lambda_{4}\left(\frac{x^{2}}{8 t}\right)^{\frac{1-2 \alpha}{2}}\left(\frac{y^{2}}{8 t}\right)^{\frac{1-2 \beta}{2}} \Psi_{2}\left(\frac{3-2 \alpha-2 \beta}{2} ; \frac{3-2 \alpha}{2}, \frac{3-2 \beta}{2} ;-\frac{x^{2}}{8 t},-\frac{y^{2}}{8 t}\right) .
\end{gathered}
$$


Substituting $\omega_{i}, i=1,2,3,4$, in (14), we obtain the following special solutions of equation (13):

$$
\begin{gathered}
u_{1}(x, y, t)=\lambda_{1} \frac{1}{\sqrt{t}} \Psi_{2}\left(\frac{1}{2} ; \frac{1+2 \alpha}{2}, \frac{1+2 \beta}{2} ;-\frac{x^{2}}{8 t},-\frac{y^{2}}{8 t}\right), \\
u_{2}(x, y, t)=\lambda_{2} \frac{x^{1-2 \alpha}}{t^{1-\alpha}} \Psi_{2}\left(1-\alpha ; \frac{3-2 \alpha}{2}, \frac{1+2 \beta}{2} ;-\frac{x^{2}}{8 t},-\frac{y^{2}}{8 t}\right), \\
u_{3}(x, y, t)=\lambda_{3} \frac{y^{1-2 \beta}}{t^{1-\beta}} \Psi_{2}\left(1-\beta ; \frac{1+2 \alpha}{2}, \frac{3-2 \beta}{2} ;-\frac{x^{2}}{8 t},-\frac{y^{2}}{8 t}\right), \\
u_{4}(x, y, t)=\lambda_{4} \frac{x^{1-2 \alpha} y^{1-2 \beta}}{t^{2-\alpha-\beta}} \Psi_{2}\left(\frac{3-2 \alpha-2 \beta}{2} ; \frac{3-2 \alpha}{2}, \frac{3-2 \beta}{2} ;-\frac{x^{2}}{8 t},-\frac{y^{2}}{8 t}\right),
\end{gathered}
$$

where $\lambda_{1}, \lambda_{2}, \lambda_{3}, \lambda_{4}$ are constants.

4. A DIFFERENTIAL EQUATION OF THE THIRD ORDER WITH ONE LINE OF DEGENERATION

Consider the equation

$$
L u \equiv y^{m} u_{x x x}-u_{y y y}=0, m=\text { const }>0,
$$

in the domain of $\Omega=\{(x, y):-\infty<x<+\infty, y>0\}$. Special solutions of equation (26) are sought in the form

$$
u=P \omega(\sigma),
$$

where

$$
P=x^{-3}, \sigma=\left(-\frac{3}{x(m+3)} y^{\frac{m+3}{3}}\right)^{3}, \beta=\frac{m}{m+3} .
$$

Substituting (27) into equation (26), we find

$$
A \omega_{\sigma \sigma \sigma}+B \omega_{\sigma \sigma}+C \omega_{\sigma}+D \omega=0,
$$

where

$$
\begin{gathered}
A=P\left(y^{m} \sigma_{x}^{3}-\sigma_{y}^{3}\right), \\
B=3\left[y^{m} P_{x} \sigma_{x}^{2}-P_{y} \sigma_{y}^{2}+P\left(y^{m} \sigma_{x} \sigma_{x x}-\sigma_{y} \sigma_{y y}\right)\right], \\
C=\left[3\left(y^{m} P_{x x} \sigma_{x}-P_{y y} \sigma_{y}\right)+3\left(y^{m} P_{x} \sigma_{x x}-P_{y} \sigma_{y y}\right)+P\left(y^{m} \sigma_{x x x}-\sigma_{y y y}\right)\right], \\
D=y^{m} P_{x x x}-P_{y y y} .
\end{gathered}
$$

After elementary calculations, we have

$$
\begin{gathered}
A=\frac{3^{3} P y^{m}}{x^{3}} \sigma^{2}(1-\sigma), \\
B=\frac{3^{3} y^{m} P}{x^{3}}\left[\frac{2+\beta}{3}+\frac{1+2 \beta}{3}+1-\left(3+1+\frac{4}{3}+\frac{5}{3}\right) \sigma\right] \sigma \\
C=\frac{3^{3} y^{m} P}{x^{3}}\left[\frac{2+\beta}{3} \frac{1+2 \beta}{3}-\left(1+1+\frac{4}{3}+\frac{5}{3}+1 \cdot \frac{4}{3}+1 \cdot \frac{5}{3}+\frac{4}{3} \cdot \frac{5}{3}\right) \sigma\right], \\
D=-\frac{3^{3} y^{m} P}{x^{3}} \cdot 1 \cdot \frac{4}{3} \cdot \frac{5}{3} .
\end{gathered}
$$


By the above equalities, from (28) it follows that

$$
\begin{aligned}
& \sigma^{2}(1-\sigma) \omega_{\sigma \sigma \sigma}+\left[\frac{2+\beta}{3}+\frac{1+2 \beta}{3}+1-\left(3+1+\frac{4}{3}+\frac{5}{3}\right) \sigma\right] \sigma \omega_{\sigma \sigma} \\
& +\left[\frac{2+\beta}{3} \frac{1+2 \beta}{3}-\left(1+1+\frac{4}{3}+\frac{5}{3}+1 \cdot \frac{4}{3}+1 \cdot \frac{5}{3}+\frac{4}{3} \cdot \frac{5}{3}\right) \sigma\right] \omega_{\sigma}-1 \cdot \frac{4}{3} \cdot \frac{5}{3} \omega=0 .
\end{aligned}
$$

Thus, we have obtained the ordinary Clausen differential equation ([27]), which has the form

$$
\begin{aligned}
& x^{2}(1-x) w_{x x x}+\left[c_{1}+c_{2}+1-\left(3+a_{1}+a_{2}+a_{3}\right) x\right] x w_{x x} \\
& +\left[c_{1} c_{2}-\left(1+a_{1}+a_{2}+a_{3}+a_{1} a_{2}+a_{1} a_{3}+a_{2} a_{3}\right) x\right] w_{x}-a_{1} a_{2} a_{3} w=0 .
\end{aligned}
$$

The Clausen equation (30) has the following three linearly independent solutions [27]:

$$
\begin{gathered}
w_{1}=\lambda_{13} F_{2}\left[\begin{array}{c}
a_{1}, a_{2}, a_{3} \\
c_{1}, c_{2}
\end{array}\right], \\
w_{2}=\lambda_{2} x^{1-c_{1}} F_{2}\left[\begin{array}{c}
a_{1}+1-c_{1}, a_{2}+1-c_{1}, a_{3}+1-c_{1} \\
2-c_{1}, c_{2}+1-c_{1}
\end{array}\right], \\
w_{3}=\lambda_{3} x^{1-c_{2}} F_{2}\left[\begin{array}{c}
a_{1}+1-c_{2}, a_{2}+1-c_{2}, a_{3}+1-c_{2} \\
c_{1}+1-c_{2}, 2-c_{2}
\end{array}\right] .
\end{gathered}
$$

Note that the Clausen function can be represented as

$$
\begin{aligned}
& { }_{3} F_{2}\left[\begin{array}{c}
a_{1}, a_{2}, a_{3} \\
c_{1}, c_{2}
\end{array}\right]=\frac{\Gamma\left(c_{1}\right) \Gamma\left(c_{2}\right)}{\Gamma\left(a_{1}\right) \Gamma\left(a_{2}\right) \Gamma\left(c_{1}-a_{1}\right) \Gamma\left(c_{2}-a_{2}\right)} \\
& \times \int_{0}^{1} \int_{0}^{1} \xi^{a_{1}-1} \eta^{a_{2}-1}(1-\xi)^{c_{1}-a_{1}-1}(1-\eta)^{c_{2}-a_{2}-1}(1-x \xi \eta)^{-a_{3}} d \xi d \eta,
\end{aligned}
$$

$\operatorname{Re} c_{i}>\operatorname{Re} a_{i}>0, i=1,2$,

$$
{ }_{3} F_{2}\left(a_{1}, a_{2}, a_{3} ; c_{1}, c_{2} ; x\right)={ }_{3} F_{2}\left[\begin{array}{c}
a_{1}, a_{2}, a_{3} \\
c_{1}, c_{2}
\end{array}\right]=\sum_{m=0}^{\infty} \frac{\left(a_{1}\right)_{m}\left(a_{2}\right)_{m}\left(a_{3}\right)_{m}}{\left(c_{1}\right)_{m}\left(c_{2}\right)_{m} m !} x^{m} .
$$

Taking into account (31) - (33), it follows from (29) that

$$
\begin{gathered}
\omega_{1}(\sigma)=\lambda_{13} F_{2}\left[\begin{array}{c}
1, \frac{4}{3}, \frac{5}{3} ; \\
\frac{2+\beta}{3}, \frac{1+2 \beta}{3} ;
\end{array}\right], \\
\omega_{2}(\sigma)=\lambda_{2} \sigma^{\frac{1-\beta}{3}}{ }_{3} F_{2}\left[\begin{array}{c}
\frac{4-\beta}{3}, \frac{5-\beta}{3}, \frac{6-\beta}{3} ; \\
\frac{4-\beta}{3}, \frac{2+\beta}{3} ;
\end{array}\right]=\lambda_{2} \sigma^{\frac{1-\beta}{3}} F\left(\frac{5-\beta}{3}, \frac{6-\beta}{3} ; \frac{2+\beta}{3} ; \sigma\right),
\end{gathered}
$$




$$
\omega_{3}(\sigma)=\lambda_{3} \sigma^{\frac{2-2 \beta}{3}}{ }_{3} F_{2}\left[\begin{array}{c}
\frac{5-2 \beta}{3}, \frac{6-2 \beta}{4-\beta}, \frac{7-2 \beta}{3}, \frac{5-2 \beta}{3} ; \\
\frac{4}{3}
\end{array}\right]=\lambda_{3} \sigma^{\frac{2-2 \beta}{3}} F\left(\frac{6-2 \beta}{3}, \frac{7-2 \beta}{3} ; \frac{4-\beta}{3} ; \sigma\right) \text {, }
$$

where $F(a, b ; c ; x)$ is the Gauss hypergeometric function ([25], [27]). Substituting (34) - (36), we finally find the following special solutions to equation (26):

$$
\begin{gathered}
u_{1}(x, y)=\lambda_{1} x^{-3}{ }_{3} F_{2}\left[\frac{1, \frac{4}{3}, \frac{5}{3}}{3}, \frac{1+2 \beta}{3} ;\left(-\frac{3}{x(m+3)} y^{\frac{m+3}{3}}\right)^{3}\right] \\
u_{2}(x, y)=\lambda_{2} x^{\beta-4} y F\left(\frac{5-\beta}{3}, \frac{6-\beta}{3} ; \frac{2+\beta}{3} ;\left(-\frac{3}{x(m+3)} y^{\frac{m+3}{3}}\right)^{3}\right), \\
u_{3}(x, y)=\lambda_{3} x^{2 \beta-5} y^{2} F\left(\frac{6-2 \beta}{3}, \frac{7-2 \beta}{3} ; \frac{4-\beta}{3} ;\left(-\frac{3}{x(m+3)} y^{\frac{m+3}{3}}\right)^{3}\right),
\end{gathered}
$$

where $\lambda_{1}, \lambda_{2}, \lambda_{3}$ are arbitrary constants.

\section{THE THIRD-ORDER DIFFERENTIAL EQUATION OF THREE VARIABLES}

In the domain $\Omega=\{(x, y, t): x>0, y>0, t>0\}$, we consider the equation

$$
L u \equiv x^{n} y^{m} u_{t}-t^{k} y^{m} u_{x x x}-t^{k} x^{n} u_{y y y}=0, m, n, k=\text { const }>0 .
$$

The solution to equation (40) is sought in the form

$$
u=P \omega(\xi, \eta),
$$

where

$$
\begin{gathered}
P=\left(\frac{2}{k+1} t^{k+1}\right)^{-1}, \xi=-\frac{k+1}{2(n+3)^{3} t^{k+1}} x^{n+3}, \eta=-\frac{k+1}{2(m+3)^{3} t^{k+1}} y^{m+3}, \\
\alpha=n /(n+3), \beta=m /(m+3) .
\end{gathered}
$$

Then substituting (41) into (40), we obtain a third-order partial differential equation

$$
A_{1} \omega_{\xi \xi \xi}+A_{2} \omega_{\eta \eta \eta}+A_{3} \omega_{\xi \xi \eta}+A_{4} \omega_{\xi \eta \eta}+A_{5} \omega_{\xi \xi}+A_{6} \omega_{\xi \eta}+A_{7} \omega_{\eta \eta}+A_{8} \omega_{\xi}+A_{9} \omega_{\eta}+A_{10} \omega=0,
$$

where

$$
\begin{gathered}
A_{1}=P t^{k}\left(y^{m} \xi_{x}^{3}+x^{n} \xi_{y}^{3}\right), A_{2}=P t^{k}\left(y^{m} \eta_{x}^{3}+x^{n} \eta_{y}^{3}\right), A_{3}=3 t^{k} P\left(y^{m} \xi_{x}^{2} \eta_{x}+x^{n} \xi_{y}^{2} \eta_{y}\right) \\
A_{4}=3 t^{k} P\left(y^{m} \xi_{x} \eta_{x}^{2}+x^{n} \xi_{y} \eta_{y}^{2}\right), A_{5}=3 t^{k}\left[y^{m} P_{x} \xi_{x}^{2}+x^{n} P_{y} \xi_{y}^{2}+P\left(y^{m} \xi_{x} \xi_{x x}+x^{n} \xi_{y} \xi_{y y}\right)\right] \\
A_{6}=3 t^{k}\left[2\left(y^{m} P_{x} \xi_{x} \eta_{x}+x^{n} P_{y} \xi_{y} \eta_{y}\right)+P\left(y^{m} \xi_{x x} \eta_{x}+x^{n} \xi_{y y} \eta_{y}\right)+P\left(y^{m} \xi_{x} \eta_{x x}+x^{n} \xi_{y} \eta_{y y}\right)\right] \\
A_{7}=3 t^{k}\left[y^{m} P_{x} \eta_{x}^{2}+x^{n} P_{y} \eta_{y}^{2}+P\left(y^{m} \eta_{x} \eta_{x x}+x^{n} \eta_{y} \eta_{y y}\right)\right] \\
A_{8}=3 t^{k}\left(y^{m} P_{x x} \xi_{x}+x^{n} P_{y y} \xi_{y}\right)+2 t^{k}\left(y^{m} P_{x} \xi_{x x}+x^{n} P_{y} \xi_{y y}\right)+t^{k}\left(y^{m} P_{x} \xi_{x x}+x^{n} P_{y} \xi_{y y}\right) \\
+t^{k} P\left(y^{m} \xi_{x x x}+x^{n} \xi_{y y y}\right)-x^{n} y^{m} P \xi_{t}
\end{gathered}
$$




$$
\begin{gathered}
A_{9}=3 t^{k}\left(y^{m} P_{x x} \eta_{x}+x^{n} P_{y y} \eta_{y}\right)+2 t^{k}\left(y^{m} P_{x} \eta_{x x}+x^{n} P_{y} \eta_{y y}\right)+t^{k}\left(y^{m} P_{x} \eta_{x x}+x^{n} P_{y} \eta_{y y}\right) \\
+t^{k} P\left(y^{m} \eta_{x x x}+x^{n} \eta_{y y y}\right)-x^{n} y^{m} P \eta_{t}, \\
A_{10}=t^{k} y^{m} P_{x x x}+t^{k} x^{n} P_{y y y}-x^{n} y^{m} P_{t} .
\end{gathered}
$$

After some calculations, we have

$$
\begin{gathered}
A_{1}=-P t^{k} x^{n} y^{m} \frac{k+1}{2 t^{k+1}} \xi^{2}, A_{2}=-P t^{k} x^{n} y^{m} \frac{k+1}{2 t^{k+1}} \eta^{2}, A_{3}=0, A_{4}=0, \\
A_{5}=-P t^{k} x^{n} y^{m}(\alpha+2) \frac{k+1}{2 t^{k+1}} \xi, A_{6}=0, A_{7}=-P t^{k} x^{n} y^{m}(2+\beta) \frac{k+1}{2 t^{k+1}} \eta, \\
A_{8}=-P t^{k} x^{n} y^{m} \frac{k+1}{2 t^{k+1}}\left(\frac{2+\alpha}{3} \frac{1+2 \alpha}{3}-2 \xi\right), A_{9}=-P t^{k} x^{n} y^{m} \frac{k+1}{2 t^{k+1}}\left(\frac{2+\beta}{3} \frac{1+2 \beta}{3}-2 \eta\right), \\
A_{10}=P t^{k} x^{n} y^{m} \frac{k+1}{t^{k+1}} .
\end{gathered}
$$

Therefore, using the indicated equalities from (42), we define

$$
\left\{\begin{array}{c}
\xi^{2} \omega_{\xi \xi \xi}+\left(\frac{2+\alpha}{3}+\frac{1+2 \alpha}{3}+1\right) \xi \omega_{\xi \xi}+\left(\frac{2+\alpha}{3} \frac{1+2 \alpha}{3}-\xi\right) \omega_{\xi}-\eta \omega_{\eta}-\omega=0 \\
\eta^{2} \omega_{\eta \eta \eta}+\left(\frac{2+\beta}{3}+\frac{1+2 \beta}{3}+1\right) \eta \omega_{\eta \eta}+\left(\frac{2+\beta}{3} \frac{1+2 \beta}{3}-\eta\right) \omega_{\eta}-\xi \omega_{\xi}-\omega=0 .
\end{array}\right.
$$

From the general theory it is easy to determine that the system of hypergeometric equations

$$
\left\{\begin{array}{l}
x^{2} w_{x x x}+\left(c_{2}+c_{1}+1\right) x w_{x x}+\left(c_{1} c_{2}-x\right) w_{x}-y w_{y}-a w=0 \\
y^{2} w_{y y y}+\left(d_{2}+d_{1}+1\right) y w_{y y}+\left(d_{1} d_{2}-y\right) w_{y}-x w_{x}-a w=0
\end{array}\right.
$$

has 9 linearly independent solutions

$$
\begin{aligned}
& w_{1}(x, y)=F_{0 ; 2 ; 2}^{1 ; 0 ; 0}\left[\begin{array}{rrr}
a ; & -; & -; \\
-; & c_{1}, c_{2} ; & d_{1}, d_{2} ;
\end{array}\right] \\
& w_{2}(x, y)=y^{1-d_{1}} F_{0 ; 2 ; 2}^{1 ; 0 ; 0}\left[\begin{array}{rrr}
1-d_{1}+a ; & -; & -; \\
-; & c_{1}, c_{2} ; & 2-d_{1}, d_{2}-d_{1}+1 ;
\end{array}\right] \text {, } \\
& w_{3}(x, y)=y^{1-d_{2}} F_{0 ; 2 ; 2}^{1 ; 0 ; 0}\left[\begin{array}{rcc}
1-d_{2}+a ; & -; & -; \\
-; & c_{1}, c_{2} ; & 2-d_{2}, d_{1}-d_{2}+1 ;
\end{array}\right] \text {, } \\
& w_{4}(x, y)=x^{1-c_{1}} F_{0 ; 2 ; 2}^{1 ; 0 ; 0}\left[\begin{array}{rrr}
1-c_{1}+a ; & -; & -; \\
-; & 2-c_{1}, c_{2}-c_{1}+1 ; & d_{1}, d_{2} ;
\end{array}\right] \text {, } \\
& w_{5}(x, y)=x^{1-c_{1}} y^{1-d_{1}} \\
& \times F_{0 ; 2 ; 2}^{1 ; 0 ; 0}\left[\begin{array}{rrr}
c_{1}+d_{1}-2-a ; & -; & -; \\
-; & 2-c_{1}, 1+c_{2}-c_{1} ; & 2-d_{1}, 1+d_{2}-d_{1} ;
\end{array}\right],
\end{aligned}
$$




$$
\begin{aligned}
& w_{6}(x, y)=x^{1-c_{1}} y^{1-d_{2}} \\
& \times F_{0 ; 2 ; 2}^{1 ; 0 ; 0}\left[\begin{array}{rrr}
c_{1}+d_{2}-2-a ; & -; & -; \\
-; & 2-c_{1}, 1+c_{2}-c_{1} ; & 1+d_{1}-d_{2}, 2-d_{2} ;
\end{array}\right], \\
& w_{7}(x, y)=x^{1-c_{2}} F_{0 ; 2 ; 2}^{1 ; 0 ; 0}\left[\begin{array}{rrr}
1-c_{2}+a ; & -; & -; \\
-; & c_{1}-c_{2}+1,2-c_{2} ; & d_{1}, d_{2} ;
\end{array}\right] \text {, } \\
& w_{8}(x, y)=x^{1-c_{2}} y^{1-d_{1}} \\
& \times F_{0 ; 2 ; 2}^{1 ; 0 ; 0}\left[\begin{array}{rrr}
c_{2}+d_{1}-2-a ; & -; & -; \\
-; & 1+c_{1}-c_{2}, 2-c_{2} ; & 2-d_{1}, 1+d_{2}-d_{1} ;
\end{array}\right] \text {, } \\
& w_{9}(x, y)=x^{1-c_{2}} y^{1-d_{2}} \\
& \times F_{0 ; 2 ; 2}^{1 ; 0 ; 0}\left[\begin{array}{rrr}
d_{2}+c_{2}-2-a ; & -; & -; x, y \\
-; & 1+c_{1}-c_{2}, 2-c_{2} ; & 1+d_{1}-d_{2}, 2-d_{2} ;
\end{array}\right] \text {, }
\end{aligned}
$$

where

$$
F_{l ; i ; j}^{p ; q ; k}\left[\begin{array}{lll}
\left(a_{p}\right) ; & \left(b_{q}\right) ; & \left(c_{k}\right) ; \\
\left(\alpha_{l}\right) ; & \left(\beta_{m}\right) ; & \left(\gamma_{n}\right) ;
\end{array}\right]=\sum_{r, s=0}^{\infty} \frac{\prod_{j=1}^{p}\left(a_{j}\right)_{r+s} \prod_{j=1}^{q}\left(b_{j}\right)_{r} \prod_{j=1}^{k}\left(c_{j}\right)_{s}}{\prod_{j=1}^{l}\left(\alpha_{j}\right)_{r+s} \prod_{j=1}^{m}\left(\beta_{j}\right)_{r} \prod_{j=1}^{n}\left(\gamma_{j}\right)_{s} r ! s !} x^{r} y^{s}
$$

are hypergeometric function of Kampe de Feriet ([27]). Then, in view of (45) - (53), the system of hypergeometric equations (43) has the following special solutions

$$
\begin{aligned}
& \omega_{1}(\xi, \eta)=F_{0 ; 2 ; 2}^{1 ; 0 ; 0}\left[\begin{array}{c}
1 ; \\
-;
\end{array} \frac{2+\alpha}{3}, \frac{1+2 \alpha}{3} ; \frac{2+\beta}{3}, \frac{1+2 \beta}{3} ; \xi, \eta\right], \\
& \omega_{2}(\xi, \eta)=\eta \frac{1-\beta}{3} F_{0 ; 2 ; 2}^{1 ; 0 ; 0}\left[\begin{array}{r}
\frac{4-\beta}{3} ; \\
-;
\end{array} \frac{2+\alpha}{3}, \frac{1+2 \alpha}{3} ; \frac{4-\beta}{3}, \frac{2+\beta}{3} ; \xi, \eta\right], \\
& \omega_{3}(\xi, \eta)=\eta \frac{2(1-\beta)}{3} F_{0 ; 2 ; 2}^{1 ; 0 ; 0}\left[\begin{array}{r}
\frac{5-2 \beta}{3} ; \\
-;
\end{array} \frac{2+\alpha}{3}, \frac{1+2 \alpha}{3} ; \frac{5-2 \beta}{3}, \frac{4-\beta}{3} ; \xi, \eta\right] \text {, } \\
& \omega_{4}(\xi, \eta)=\xi^{\frac{1-\alpha}{3}} F_{0 ; 2 ; 2}^{1 ; 0 ; 0}\left[\begin{array}{c}
\frac{4-\alpha}{3} ; \\
-;
\end{array} \frac{4-\alpha}{3}, \frac{2+\alpha}{3} ; \frac{2+\beta}{3}, \frac{1+2 \beta}{3} ; \xi, \eta\right], \\
& \omega_{5}(\xi, \eta)=\xi^{\frac{1-\alpha}{3}} \eta \frac{1-\beta}{3} F_{0 ; 2 ; 2}^{1 ; 0 ; 0}\left[\begin{array}{r}
\frac{\alpha+\beta-5}{3} ; \\
-;
\end{array} \frac{4-\alpha}{3}, \frac{2+\alpha}{3} ; \frac{4-\beta}{3}, \frac{2+\beta}{3} ; \xi, \eta\right],
\end{aligned}
$$




$$
\begin{aligned}
& \omega_{6}(\xi, \eta)=\xi \frac{1-\alpha}{3} \eta \frac{2(1-\beta)}{3} F_{0 ; 2 ; 2}^{1 ; 0 ; 0}\left[\begin{array}{r}
\frac{\alpha+2 \beta-6}{3} ; \\
-;
\end{array} \frac{4-\alpha}{3}, \frac{2+\alpha}{3} ; \frac{4-\beta}{3}, \frac{5-2 \beta}{3} ; \xi, \eta\right] \\
& \omega_{7}(\xi, \eta)=\xi^{\frac{2(1-\alpha)}{3}} F_{0 ; 2 ; 2}^{1 ; 0 ; 0}\left[\begin{array}{r}
\frac{5-2 \alpha}{3} ; \\
-;
\end{array} \frac{4-\alpha}{3}, \frac{5-2 \alpha}{3} ; \frac{2+\beta}{3}, \frac{1+2 \beta}{3} ; \xi, \eta\right] \\
& \omega_{8}(\xi, \eta)=\xi^{\frac{2(1-\alpha)}{3}} \eta^{\frac{1-\beta}{3}} F_{0 ; 2 ; 2}^{1 ; 0 ; 0}\left[\begin{array}{r}
\frac{2 \alpha+\beta-6}{3} ; \\
-\frac{4-\alpha}{3}, \frac{5-2 \alpha}{3} ;
\end{array} \frac{4-\beta}{3}, \frac{2+\beta}{3} ; \xi, \eta\right] \\
& \omega_{9}(\xi, \eta)=\xi^{\frac{2(1-\alpha)}{3}} \eta^{\frac{2(1-\beta)}{3}} F_{0 ; 2 ; 2}^{1 ; 0 ; 0}\left[\begin{array}{r}
\frac{2 \alpha+2 \beta-7}{3} ; \\
-;
\end{array} \frac{4-\alpha}{3}, \frac{5-2 \alpha}{3} ; \frac{4-\beta}{3}, \frac{5-2 \beta}{3} ; \xi, \eta\right] .
\end{aligned}
$$

Multiplying each solution by $P=\left(\frac{2}{k+1} t^{k+1}\right)^{-1}$, we finally get special solutions for equation $(40)$ :

$$
\begin{aligned}
& u_{1}(x, y, t)=\lambda_{1} P F_{0 ; 2 ; 2}^{1 ; 0 ; 0}\left[\begin{array}{c}
1 ; \\
-;
\end{array} \frac{2+\alpha}{3}, \frac{1+2 \alpha}{3} ; \frac{2+\beta}{3}, \frac{1+2 \beta}{3} ; \xi, \eta\right] \\
& u_{2}(x, y, t)=\lambda_{2} P \eta \frac{1-\beta}{3} F_{0 ; 2 ; 2}^{1 ; 0 ; 0}\left[\begin{array}{c}
\frac{4-\beta}{3} ; \\
-;
\end{array} \frac{2+\alpha}{3}, \frac{1+2 \alpha}{3} ; \frac{4-\beta}{3}, \frac{2+\beta}{3} ; \xi, \eta\right] \\
& u_{3}(x, y, t)=\lambda_{3} P \eta \frac{2(1-\beta)}{3} F_{0 ; 2 ; 2}^{1 ; 0 ; 0}\left[\begin{array}{r}
\frac{5-2 \beta}{3} ; \\
-;
\end{array} \frac{2+\alpha}{3}, \frac{1+2 \alpha}{3} ; \frac{5-2 \beta}{3}, \frac{4-\beta}{3} ; \xi, \eta\right] \text {, } \\
& u_{4}(x, y, t)=\lambda_{4} P \xi^{\frac{1-\alpha}{3}} F_{0 ; 2 ; 2}^{1 ; 0 ; 0}\left[\begin{array}{r}
\frac{4-\alpha}{3} ; \\
-;
\end{array} \frac{4-\alpha}{3}, \frac{2+\alpha}{3} ; \frac{2+\beta}{3}, \frac{1+2 \beta}{3} ; \xi, \eta\right] \\
& u_{5}(x, y, t)=\lambda_{5} P \xi^{\frac{1-\alpha}{3}} \eta^{\frac{1-\beta}{3}} F_{0 ; 2 ; 2}^{1 ; 0 ; 0}\left[\begin{array}{r}
\frac{\alpha+\beta-5}{3} ; \\
-;
\end{array} \frac{4-\alpha}{3}, \frac{2+\alpha}{3} ; \frac{4-\beta}{3}, \frac{2+\beta}{3} ; \xi, \eta\right], \\
& u_{6}(x, y, t)=\lambda_{6} P \xi \frac{1-\alpha}{3} \eta \frac{2(1-\beta)}{3} F_{0 ; 2 ; 2}^{1 ; 0 ; 0}\left[\begin{array}{r}
\frac{\alpha+2 \beta-6}{3} ; \\
-;
\end{array} \frac{4-\alpha}{3}, \frac{2+\alpha}{3} ; \frac{4-\beta}{3}, \frac{5-2 \beta}{3} ; \xi, \eta\right] \\
& u_{7}(x, y, t)=\lambda_{7} P \xi^{\frac{2(1-\alpha)}{3}} F_{0 ; 2 ; 2}^{1 ; 0 ; 0}\left[\begin{array}{r}
\frac{5-2 \alpha}{3} ; \\
-;
\end{array} \frac{4-\alpha}{3}, \frac{5-2 \alpha}{3} ; \frac{2+\beta}{3}, \frac{1+2 \beta}{3} ; \xi, \eta\right] \text {, }
\end{aligned}
$$




$$
\begin{aligned}
& u_{8}(x, y, t)=\lambda_{8} P \xi^{\frac{2(1-\alpha)}{3}} \eta \frac{1-\beta}{3} F_{0 ; 2 ; 2}^{1 ; 0 ; 0}\left[\begin{array}{r}
\frac{2 \alpha+\beta-6}{3} ; \\
-;
\end{array} \frac{4-\alpha}{3}, \frac{5-2 \alpha}{3} ; \frac{4-\beta}{3}, \frac{2+\beta}{3} ; \xi, \eta\right] \text {, } \\
& u_{9}(x, y, t)=\lambda_{9} P \xi \frac{2(1-\alpha)}{3} \eta \frac{2(1-\beta)}{3} F_{0 ; 2 ; 2}^{1 ; 0 ; 0}\left[\begin{array}{r}
\frac{2 \alpha+2 \beta-7}{3} ; \\
-;
\end{array} \frac{-;}{3}, \frac{4-\alpha}{3} ; \frac{4-\beta}{3}, \frac{5-2 \beta}{3} ; \xi, \eta\right] \text {, }
\end{aligned}
$$

where $\lambda_{1}, \ldots, \lambda_{9}$ are constants.

\section{A FOURTH-ORDER DIFFERENTIAL EQUATION WITH TWO LINES OF DEGENERACY}

In the domain $\Omega=\{(x, t): x>0, t>0\}$, we consider the equation

$$
L u \equiv x^{n} u_{t}-t^{k} u_{x x x x}=0, n, k=\text { const }>0 .
$$

Special solutions of equation (64) will be sought in the form

$$
u(x, t)=P(t) \omega(\sigma),
$$

where

$$
P=\left(\frac{1}{k+1} t^{k+1}\right)^{-1}, \quad \sigma=-\frac{k+1}{(n+4)^{4} t^{k+1}} x^{n+4} .
$$

Substituting (65) into equation (64), we define

$$
\begin{aligned}
& t^{k} P \omega_{\sigma \sigma \sigma \sigma} \sigma_{x}^{4}+6 t^{k} P \sigma_{x x} \sigma_{x}^{2} \omega_{\sigma \sigma \sigma} \\
& +t^{k}\left[3 P \sigma_{x x}^{2}+4 P \sigma_{x} \sigma_{x x x}\right] \omega_{\sigma \sigma}+\left[t^{k} P \sigma_{x x x x}-x^{n} P \sigma_{t}\right] \omega_{\sigma}-x^{n} P_{t} \omega=0 .
\end{aligned}
$$

After some calculations, we have

$$
\begin{aligned}
& \sigma^{3} \omega_{\sigma \sigma \sigma \sigma}+\left(3+\frac{3+\alpha}{4}+\frac{2+2 \alpha}{4}+\frac{1+3 \alpha}{4}\right) \sigma^{2} \omega_{\sigma \sigma \sigma}+\left(1+\frac{3+\alpha}{4}+\frac{2+2 \alpha}{4}+\frac{1+3 \alpha}{4}\right. \\
& \left.+\frac{3+\alpha}{4} \frac{2+2 \alpha}{4}+\frac{3+\alpha}{4} \frac{1+3 \alpha}{4}+\frac{2+2 \alpha}{4} \frac{1+3 \alpha}{4}\right) \sigma \omega_{\sigma \sigma} \\
& +\left(\frac{3+\alpha}{4} \frac{2+2 \alpha}{4} \frac{1+3 \alpha}{4}-\sigma\right) \omega_{\sigma}-\omega=0,
\end{aligned}
$$

where $\alpha=n /(n+4)$. From the general theory ([27]) it is known that the equation

$$
\begin{aligned}
& x^{3} w_{x x x x}+\left(3+c_{1}+c_{2}+c_{3}\right) x^{2} w_{x x x}+ \\
& +\left(1+c_{1}+c_{2}+c_{3}+c_{1} c_{2}+c_{1} c_{3}+c_{2} c_{3}\right) x w_{x x}+\left(c_{1} c_{2} c_{3}-x\right) w_{x}-a w=0,
\end{aligned}
$$

has the following special solutions

$$
\begin{gathered}
w_{1}=\lambda_{11} F_{3}\left(a ; c_{1}, c_{2}, c_{3} ; x\right), \\
w_{2}=\lambda_{2} x^{1-c_{1}}{ }_{1} F_{3}\left(1-c_{1}+a ; 2-c_{1}, 1+c_{2}-c_{1}, 1+c_{3}-c_{1} ; x\right), \\
w_{3}=\lambda_{3} x^{1-c_{2}}{ }_{1} F_{3}\left(1-c_{2}+a ; 1+c_{1}-c_{2}, 2-c_{2}, 1+c_{3}-c_{2} ; x\right), \\
w_{4}=\lambda_{4} x^{1-c_{3}}{ }_{1} F_{3}\left(1-c_{3}+a ; 1+c_{1}-c_{3}, 1+c_{2}-c_{3}, 2-c_{3} ; x\right),
\end{gathered}
$$


where $\lambda_{i}$ are constants, $i=1,2,3,4$, and

$$
{ }_{1} F_{3}\left(a ; c_{1}, c_{2}, c_{3} ; x\right)=\sum_{m=0}^{\infty} \frac{(a)_{m}}{\left(c_{1}\right)_{m}\left(c_{2}\right)_{m}\left(c_{3}\right)_{m} m !} x^{m} .
$$

Then from equation (67), in view of (69) - (72), taking into account representation (65), it is easy to determine special solutions to equation (64):

$$
\begin{gathered}
u_{1}(x, t)=\bar{\lambda}_{1}\left(\frac{1}{k+1} t^{k+1}\right)^{-1}{ }_{1} F_{3}\left(1 ; \frac{3+\alpha}{4}, \frac{2+2 \alpha}{4}, \frac{1+3 \alpha}{4} ;-\frac{k+1}{(n+4)^{4} t^{k+1}} x^{n+4}\right), \\
u_{2}(x, t)=\bar{\lambda}_{2}\left(\frac{1}{k+1} t^{k+1}\right)^{-\frac{9-\alpha}{4}} x_{0} F_{2}\left(\frac{3+\alpha}{4}, \frac{2+2 \alpha}{4} ;-\frac{k+1}{(n+4)^{4} t^{k+1}} x^{n+4}\right), \\
u_{3}(x, t)=\bar{\lambda}_{3}\left(\frac{1}{k+1} t^{k+1}\right)^{-\frac{6-2 \alpha}{4}} x_{0}^{2} F_{2}\left(\frac{5-\alpha}{4}, \frac{3+\alpha}{4} ;-\frac{k+1}{(n+4)^{4} t^{k+1}} x^{n+4}\right), \\
u_{4}(x, t)=\bar{\lambda}_{4}\left(\frac{1}{k+1} t^{k+1}\right)^{-\frac{11-3 \alpha}{4}} x_{0}{ }_{0} F_{2}\left(\frac{6-2 \alpha}{4}, \frac{5-\alpha}{4} ;-\frac{k+1}{(n+4)^{4} t^{k+1}} x^{n+4}\right),
\end{gathered}
$$

where $\bar{\lambda}_{i}$ are constants, $i=1,2,3,4$.

\section{REFERENCES}

1. L. I. Sedov, Similarity and dimension methods in mechanics (Science, Moscow, 1977). (in Russian)

2. L. D. Landau and E. M. Lifshits, "Investigation of the flow features using the Euler-Tricomi equation," DAN AN USSR, 96, 725-728 (1954), (in Russian).

3. G. Lohofer, "Theory of an electromagnetically deviated metal sphere. I: Absorbed power," SIAM J. Appl. Math. 49, 567B-581 (1989).

4. A. W. Niukkanen,"Generalised hypergeometric series ${ }^{N} F\left(x_{1}, \ldots, x_{N}\right)$ arising in physical and quantum chemical applications," J. Phys. A: Math. Gen. 16, 1813-1825 (1983).

5. R Courant, K. Friedrichs, Supersonic flow and shock waves (New York, 1984).

6. L. Bers, Mathematical problems of subsonic and transonic gas dynamics (M., IL., 1961). (in Russian).

7. F. I. Frankl, Selected Works in Gas Dynamics (Nauka, Moscow, 1973) (in Russian).

8. T. G. Ergashev, A. Hasanov, "Fundamental solutions of the bi-axially symmetric Helmholtz equation," Uzbek Mathematical Journal 1, 55-64 (2018).

9. R. B. Seilkhanova, A. Hasanov, "Particular solutions of generalized Euler-Poisson- Darboux equation," Electron. J. Diff. Equ. 9, 1-10 (2015).

10. A. Hasanov, J. M. Rassias and M. Turaev, Functional Equations, difference Inequalities and ULAM Stability Notions, Fundamental solution for the generalized Elliptic Gellerstedt Equation (Nova Science Publishers Inc. NY, USA, 2010), 6, 73-83.

11. A. Hasanov and E. T. Karimov,"Fundamental solutions for a class of three-dimensional elliptic equations with singular coefficients," Applied Mathematic Letters 22, 1828-1832 (2009).

12. M. S. Salakhitdinov and A. Hasanov, "The Fundamental solution for one class of degenerate elliptic equations," More Progresses in Analysis, Proceedings of the 5-th International ISAAC Congress. World Scientific Publishing Co Pte. Ltd., 521-531 (2009).

13. A. Hasanov,"Fundamental solutions for degenerated elliptic equation with two perpendicular lines of degeneration," International J. of Applied Mathematics and Statistics 8 13, 41-49 (2008).

14. M. S. Salakhitdinov, A. Hasanov, "To the theory of the multidimensional Gellerstedt equation," Uzbek Mathematical Journal 3, 95-109 (2007). (in Russian)

15. A. Hasanov,"Fundamental solutions bi-axially symmetric Helmholtz equation," Complex Variables and Elliptic Equations 52 (8), 673-683 (2007).

16. A. Hasanov,"Some solutions of generalized RassiasB's equation," International J. of Applied Mathematics and Statistics 8 M07, 20-30 (2007).

17. A. Hasanov,"Fundamental Solutions of two Degenerated Elliptic Equations and Solutions of Boundary Value Problems in Infinite Area," International J. of Applied Mathematics and Statistics 8 M07, 87-95 (2007). 
18. A. Hasanov,"Fundamental solutions of generalized Helmholtz equation," Reports of Uzbek Academy of Sciences 1, 13-15 (2006).

19. T. G. Ergashev, A. Hasanov,"Fundamental solutions of the bi-axially symmetric Helmholtz equation," Uzbek Mathematical Journal 1, 55-64 (2018).

20. H. M. Srivastava, A. Hasanov and Junesang Choi,"Double-Layer Potentials for a Generalized Bi-Axially Symmetric Helmholtz Equation," Sohag Journal of Mathematics An International Journal 1, 2, 1-10 (2015).

21. M. S. Salakhitdinov and A. Hasanov,"The Dirichlet problem for the generalized bi-axially symmetric Helmholtz equation," Eurasian Mathematical Journal 3, 4, 99-110 (2012).

22. M. S. Salakhitdinov M. S. and A. Hasanov,"A solution of the Neumann-Dirichlet boundary value problem for generalized bi-axially symmetric Helmholtz equation," Complex Variables and Elliptic Equations 4, $53,355-364$ (2008).

23. A. Hasanov,"The solution of the Cauchy problem for generalized Euler-Poisson-Darboux equation," International J. of Applied Mathematics and Statistics 8 M07, 30-44 (2007).

24. M. S. Salakhitdinov and A. Hasanov,"The Tricomi problem for an equation of mixed type with a no smooth line of degeneracy," DifferentsialB'nye Uravneniya 1 19, 110-119 (1983).

25. A. Erdélyi, W. Magnus, F. Oberhettinger, and F. G. Tricomi, Higher Transcendental Functions. 1, McGraw-Hill, New York, Toronto and London, (1953).

26. Sh. Karimov,"On one method for the solution of an analog of the Cauchy problem for a polycaloric equation with singular Bessel operator," Ukrainian Mathematical Journal 69 10, 1593-1606 (1918).

27. P. Appell and J. Kampé de Fériet, Fonctions Hypergeometriques et Hyperspheriques; Polynomes d'Hermite (Gauthier - Villars, Paris, 1926). 